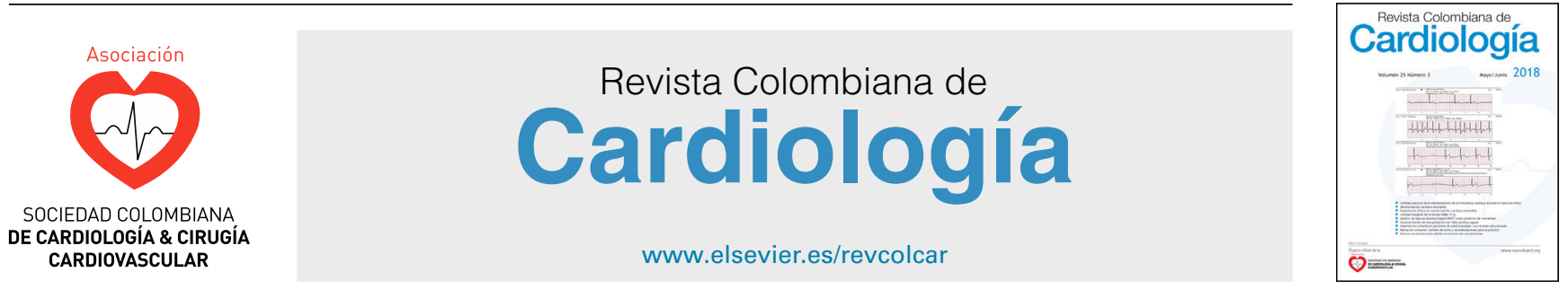

INNOVACIÓN EN CARDIOLOGÍA

\title{
Implante experimental de una bomba de asistencia circulatoria de flujo continuo en posición valvular aórtica
}

\author{
Enrique Seguel ${ }^{\mathrm{a}, \mathrm{b}, *}$, Sylvain Beurtherert ${ }^{\mathrm{c}}$, Daniel Herzberg ${ }^{\mathrm{a}, \mathrm{d}}$, Luis Gutiérrez ${ }^{\mathrm{a}, \mathrm{d}}$, \\ Sergio Cofréa ${ }^{a, d}$ y Alan Labra ${ }^{a, d}$
}

a Grupo de Investigaciones Quirúrgicas, Universidad de Concepción, Chile

b Departamento de Cirugía, Facultad de Medicina, Universidad de Concepción, Chile

c Servicio de Cirugía Cardiaca Adulto, Hospital Saint Joseph, Marsella, Francia

d Departamento de Ciencias Clínicas, Facultad de Ciencias Veterinarias, Universidad de Concepción, Chile

Recibido el 18 de julio de 2017; aceptado el 6 de agosto de 2018

Disponible en Internet el 25 de marzo de 2019

\author{
PALABRAS CLAVE \\ Dispositivo de \\ asistencia cardiaca; \\ Modelos animales; \\ Insuficiencia \\ cardiaca; \\ Cirugía cardiaca; \\ Bioimplantes; \\ Jarvik 2000
}

\begin{abstract}
Resumen
Introducción: el implante de una bomba de flujo axial en posición valvular aórtica podría disminuir ciertas complicaciones de las bombas actuales, permitiría un flujo sanguíneo anterógrado en el corazón y la aorta, evitaría la confección de un bolsillo, omitiría la necesidad de cánulas de entrada y salida, e impediría la formación de cicatriz en la punta del corazón.

Objetivo: estudiar la factibilidad técnica del implante de una bomba de flujo axial en posición valvular aórtica, además de evaluar los efectos hemodinámicos y su impacto sobre las estructuras cardiacas.

Métodos: se implantó una bomba Jarvik $2000^{\oplus}$ adaptada para implante intravalvular en 7 cerdos mediante toracotomía anterior izquierda bajo circulación extracorpórea. La bomba se fijó al anillo aórtico mediante suturas. Se asistió a los sujetos durante una hora y se evaluaron el comportamiento hemodinámico y las estructuras cardiacas con ecocardiograma epicárdico. Luego de la eutanasia del animal se explantó el corazón para revisión de las cavidades.

Resultados: todos los procedimientos se completaron con la técnica. Con apoyo de drogas vasoactivas y marcapasos epicárdico, la bomba fue capaz de mantener la hemodinamia de los individuos. El ecocardiograma mostró llenado adecuado del ventrículo izquierdo y buena función de la válvula mitral. El examen postmortem mostró que los ostia coronarios estaban libres y no había trombos en las cavidades cardiacas.
\end{abstract}

\footnotetext{
* Autor para correspondencia.

Correo electrónico: enseguel@udec.cl (E. Seguel).
} 
Conclusiones: en este modelo animal fue posible el implante de una bomba de flujo axial en posición valvular aórtica. La bomba pudo mantener la hemodinamia del animal sin afectar significativamente las estructuras cardiacas.

(C) 2019 Sociedad Colombiana de Cardiología y Cirugía Cardiovascular. Publicado por Elsevier España, S.L.U. Este es un artículo Open Access bajo la licencia CC BY-NC-ND (http:// creativecommons.org/licenses/by-nc-nd/4.0/).

\section{KEYWORDS}

Cardiac assist device; Animal models; Heart failure; Cardiac surgery; Bio-implants; Jarvik 2000

\section{Experimental implant of a continuous flow circulation pump in an aortic valve position}

\begin{abstract}
Introduction: The implanting of an axial flow pump in an aortic valve position could decrease certain complications in current pumps. It could permit an anterograde blood flow in the heart and the aorta. It could avoid the making of a bag, as well as avoiding the need for inlet and outlet cannulas, and could slow down the scar formation in the cardiac apex.

Objective: To study the technical feasibility of the implanting of an axial flow pump in an aortic valve position, as well as to assess the haemodynamic effects and its impact on cardiac structures.

Methods: A Jarvik $2000^{\oplus}$ pump adapted for an intra-valvular implant in 7 pigs was inserted using left anterior thoracotomy and under extracorporeal circulation. The pump was fixed to the aortic ring using sutures. It assisted the subjects for an hour during an assessment was made of the haemodynamic behaviour and the cardiac structures with an epicardial echocardiogram. After euthanasia of the animal, the heart was removed for an examination of its cavities.

Results: All the procedures were completed with the technique. With the vasoactive drugs and epicardial pacemaker support, the pump was capable of maintaining the haemodynamics of the individuals. The echocardiogram showed adequate filling of the left ventricle and good functioning of the mitral valve. The post-mortem examination showed that the coronary ostia were free and there had not been any clots in the cardiac cavities.

Conclusions: In this animal model, the implanting of an axial flow pump in an aortic valve position was possible. The pump was able to maintain the haemodynamics of the animal without significantly affecting the cardiac structures.

(C) 2019 Sociedad Colombiana de Cardiología y Cirugía Cardiovascular. Published by Elsevier España, S.L.U. This is an open access article under the CC BY-NC-ND license (http:// creativecommons.org/licenses/by-nc-nd/4.0/).
\end{abstract}

\section{Introducción}

Las bombas de asistencia circulatoria de flujo axial se han convertido en los sistemas de asistencia más utilizados en la actualidad para los pacientes portadores de insuficiencia cardiaca en etapa terminal. Entre sus ventajas están su pequeño tamaño, menor riesgo de trombosis, y su mayor eficiencia energética respecto a las bombas de flujo pulsátil. Los resultados del estudio Heartmate, que comparó el uso de una bomba neumática con una bomba de flujo continuo, mostraron que la supervivencia a los dos años del grupo que recibió la bomba neumática fue de 24 versus $58 \%$ de aquellos tratados con la bomba de flujo continuo $(p 0,008)^{1}$. Estos resultados confirmaron que, además de sus ventajas técnicas, existe una ventaja en la supervivencia para los pacientes con el uso de este tipo de dispositivos.

A pesar de los avances técnicos en el desarrollo de estos dispositivos, aún persiste una tasa de complicaciones asociadas a éstos.

En diversos reportes se han descrito problemas anatómicos, asociados a la confección del bolsillo; problemas relacionados con la posición de la cánula de entrada, así como complicaciones con la cánula de salida. El implante se relaciona con una tasa de hemorragias postoperatorias descrita de entre un 0,16 hasta 2,45 eventos por paciente al año. Pese al uso de anticoagulación, la tasa de eventos trombóticos y embolias alcanza un $12 \%$. Así mismo, se describe una tasa de infecciones asociadas al dispositivo cercana al $12 \%$, e incluye las infecciones del dispositivo, de la línea y a distancia. Debido a que la válvula aórtica permanece cerrada y a que el flujo a nivel de la aorta ascendente es retrógrado, se ha descrito la fusión de las comisuras valvulares y la formación de trombos en la válvula ${ }^{2-12}$.

Finalmente, frente a reoperación por alguna complicación, para realizar un trasplante o para el retiro de la asistencia en caso de recuperación, las fuertes adherencias pericárdicas dificultan la cirugía e incrementan el riesgo de sangrado.

La idea de crear una bomba ubicada en la posición de la válvula aórtica (valvo - pump) fue publicada por primera vez por Yoshinori Mitamura en $1991^{13}$. El implante en esta posición, de forma similar a un reemplazo valvular aórtico, permitiría un flujo sanguíneo anterógrado en el corazón y la aorta, evitaría la confección del bolsillo, omitiría la necesi- 
dad de cánulas de entrada y salida, remediaría la formación de cicatriz en la punta del corazón y podría disminuir algunas de las complicaciones asociadas con estos sistemas.

Por el momento no existe una bomba diseñada para este tipo de implante y se desconocen los efectos hemodinámicos y sobre las estructuras cardiacas. Los prototipos de la valvopump publicados hasta hoy, están en una etapa experimental y no están adaptados para uso en periodos prolongados o en humanos ${ }^{14-21}$.

Las hipótesis de trabajo fueron las siguientes:

- El implante de una bomba de flujo axial en posición valvular aórtica en un animal de experimentación es técnicamente posible.

- El anillo valvular es capaz de sostener la bomba.

- La bomba implantada en esta posición podrá sostener la hemodinamia del animal.

- Las estructuras cardiacas no se verán afectadas de manera significativa con la bomba in situ.

\section{Material y métodos}

El protocolo del estudio fue aprobado por el comité de Bioética de la Facultad de Ciencias Veterinarias de la Universidad de Concepción, dentro del Proyecto Dirección Investigación Universidad de Concepción № 211.081.028-1,0; Universidad de Concepción, Concepción, Chile.

\section{Centros de investigación}

Tres de las experiencias fueron realizadas en el IMM Recherche, Preclinical research - surgical training - GLP studies, Institut Mutualiste Montsouris, Paris, Francia. Otras cuatro fueron realizadas en la Clínica Veterinaria de la Universidad de Concepción, Concepción, Chile.

\section{Bomba}

Para el implante se eligió el Jarvik $2000^{\circledR}$ (Jarvik Heart, Inc, New York, NY) ya que es una bomba de flujo axial diseñada para ser implantada en posición intraventricular, sin necesidad de una cánula de entrada.

Para su adaptación se procedió al retiro del tubo de salida, al retiro de la cubierta rugosa y a la instalación de un anillo de dacrón para su fijación al anillo aórtico (fig. 1).

Para evaluar la factibilidad anatómica se procedió al implante ex-vivo de la bomba en la posición deseada en corazones frescos de cerdo. Debido a que el diámetro de la bomba es $27 \mathrm{~mm}$ se debieron utilizar corazones de cerdos de más de $100 \mathrm{~kg}$. Una vez completado el procedimiento se observó que las arterias coronarias quedaron libres. Al abrir el corazón se observó que la cavidad ventricular quedó libre y que el velo anterior de la mitral fue empujado hacia atrás, pero sin ocluir el orificio de la válvula (figs. 2 y 3 ).

\section{Modelo animal}

Se utilizaron 8 cerdos domésticos mestizos hembras de 6 meses de edad y de más de $100 \mathrm{~kg}$ de peso. Un cerdo fallece en la inducción anestésica y no se realizó el implante.

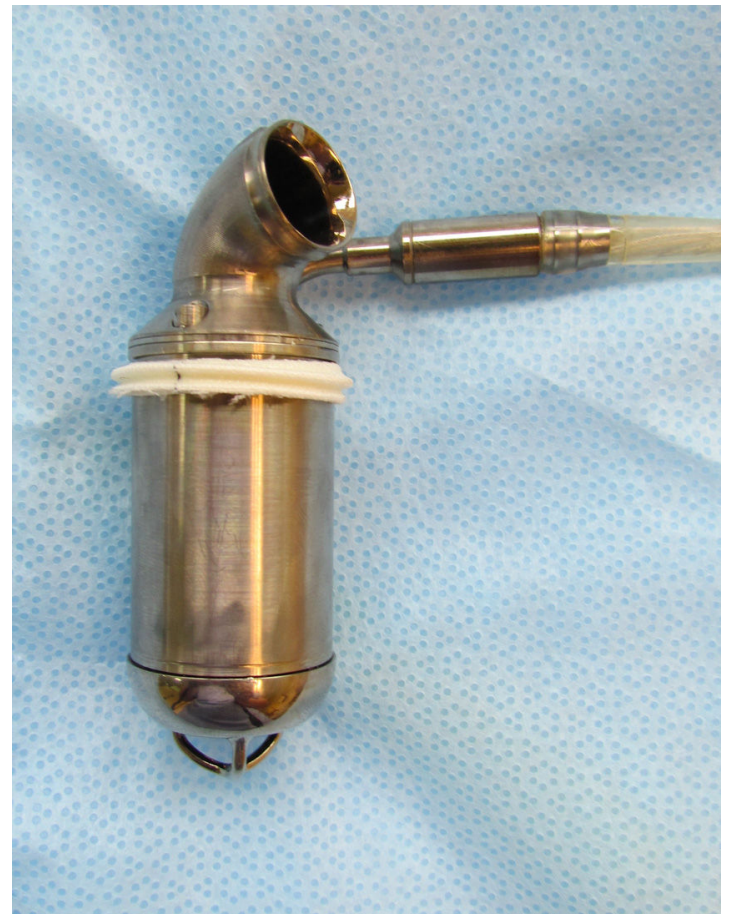

Figura 1

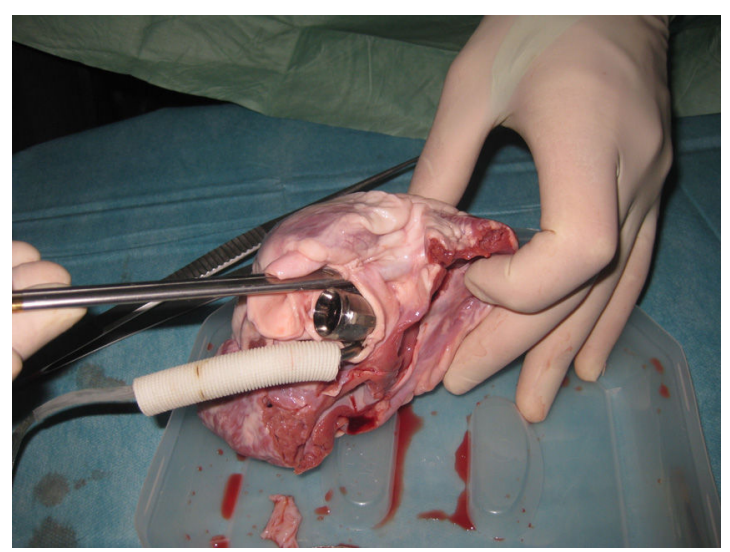

Figura 2

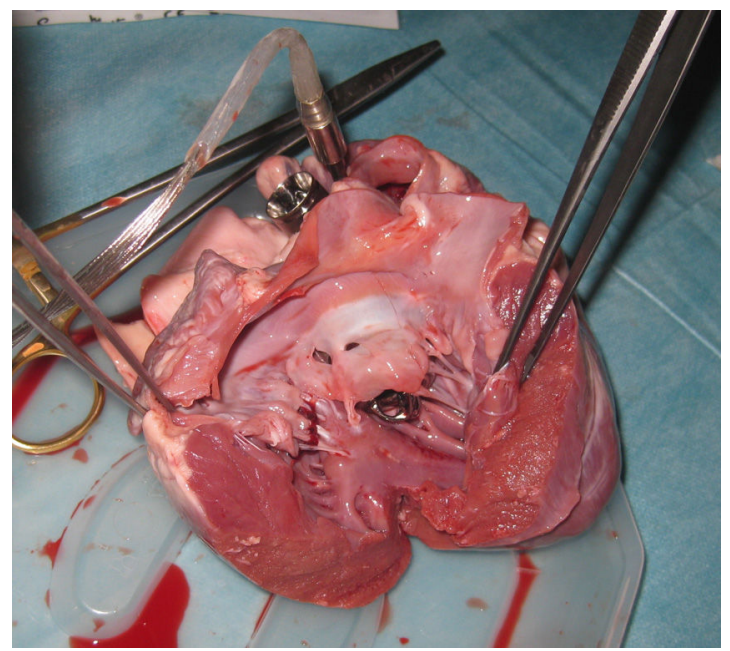

Figura 3 
Los sujetos fueron tratados en cumplimiento de la Ley 20.380, de 2010, del Gobierno de Chile sobre Protección de Animales y a la Directiva 2012 / 63 / UE del Parlamento Europeo y el Consejo de protección de animales usados para fines científicos, de septiembre de $2010^{22,23}$.

\section{Protocolo de anestesia}

La premedicación consistió en la administración intramuscular de una combinación de $2 \mathrm{mg} / \mathrm{kg}$ de azaperona (Stresnil $^{\circledR}$, Laboratorio Drag Pharma) y $50 \mathrm{mg} / \mathrm{kg}$ de ketamina (Ketamil ${ }^{\circledR}$, Laboratorio Troy). Se obtuvo una vía venosa auricular para administración de solución de lactato de Ringer a $10 \mathrm{ml} / \mathrm{kg} /$ hora, e inducción anestésica combinada utilizando un cono nasal con isoflurano (Isoflurano, Laboratorio Baxter) y 2 - $4 \mathrm{mg} / \mathrm{kg}$ de propofol (Diprofol ${ }^{\circledR}$, Laboratorio Bharat) por vía intravenosa (IV). Tras la estabilización del plano anestésico se procede a la intubación oro traqueal con tubo endotraqueal de 8,5 - 9,5. Los sujetos fueron ventilados a presión positiva intermitente utilizando un volumen tidal de $15 \mathrm{~mL} / \mathrm{kg}$, una razón inspiración:espiración de $1: 2$ y frecuencia respiratoria de 8 a 15 ciclos por minuto, ajustada para un $\mathrm{CO}_{2}$ espirado entre 35 - $45 \mathrm{~mm} \mathrm{Hg}$. El sostenimiento de la anestesia se realizó con una mezcla de $\mathrm{O}_{2}$ al $100 \%$ e isoflurano, ajustando el vaporizador para lograr una concentración de isoflurano de $1,7 \%$.

\section{Monitorización}

Se introdujo un catéter venoso central vía yugular derecha para medición de presión venosa central y aporte de drogas vasoactivas. Se instaló una línea arterial por denudación de la arteria femoral izquierda y se realizó sondeo urinario mediante una sonda Foley implantada directamente en la vejiga por vía abdominal.

Se monitorizaron los signos vitales con electrocardiograma, saturación de $\mathrm{O}_{2}$ mediante oximetría de pulso conectado en la oreja, presión arterial invasiva sistémica, tasa espirada de $\mathrm{CO}_{2}$ mediante capnografía y temperatura mediante una sonda transesofágica.

\section{Técnica quirúrgica}

El abordaje y la exposición del corazón se llevaron a cabo por toracotomía anterior amplia en el cuarto espacio intercostal izquierdo. Una vez expuesto el corazón se procedió a la obtención de imágenes de ecocardiografía epicárdica basal mediante un ecógrafo Mindrive DC - 6 vet $^{\circledR}$ (Shenzhen Mindray Bio - medical Electronics Co. Ltd. China) y un transductor sectorial de $3,5 \mathrm{MHz}$ modelos $6 \mathrm{C} 2$ y $2 \mathrm{P} 2$.

De manera simultánea a la realización del ecocardiograma se disecó la arteria femoral derecha para la canulación arterial.

Se procedió a la anticoagulación plena mediante heparina endovenosa $(5 \mathrm{mg} / \mathrm{kg})$. Confección de jaretas y canulación arterial en arteria femoral utilizando cánula femoral de 18 french, canulación venosa en orejuela derecha con cánula venosa de una etapa de 32 french y vent en arteria pulmonar con cánula angulada de 20 french. Entrada en circulación extracorpórea con bomba de rodillo (Sans $7000^{\circledR}$, Sarns Inc.

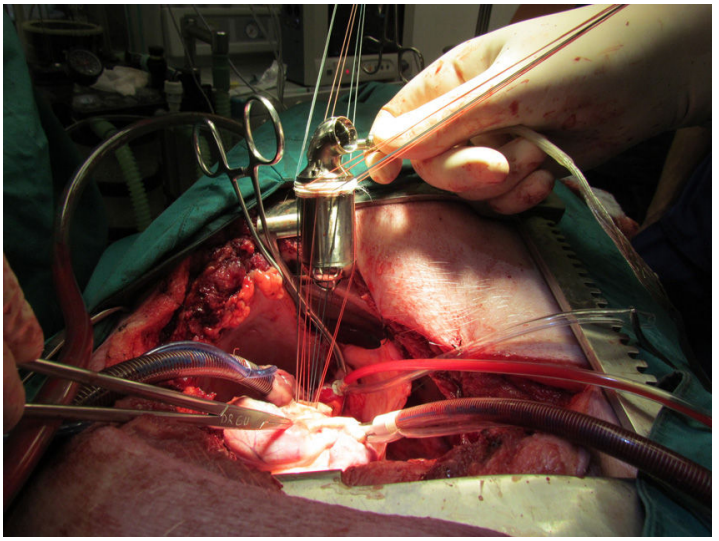

Figura 4

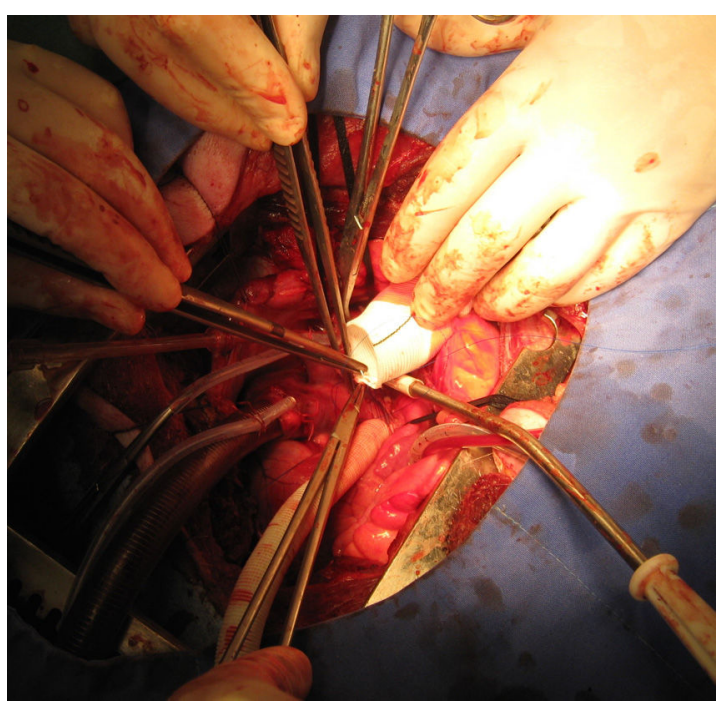

Figura 5

Ann Arbor, MI, USA) y oxigenador de membrana (Affinity ${ }^{\circledR} \mathrm{NT}$ Oxigenator, Medtronics). La perfusión se mantuvo a flujos entre 4 y $5 \mathrm{~L} / \mathrm{min}$ para mantener una presión arterial media de $50 \mathrm{mmHg}$.

Una vez estabilizada la circulación extracorpórea se procedió al pinzamiento de la aorta y a la detención del corazón utilizando una solución de cardioplejía cristaloide fría $(500 \mathrm{ml}$ de solución salina al $90 / 00+2 \mathrm{~g}$ de $\mathrm{KCl}+2,5 \mathrm{~g}$ de $\mathrm{Mg} \mathrm{SO}_{4}$ ) mediante una aguja en la aorta ascendente (vía anterógrada).

Una vez detenido el corazón se realizó aortotomía transversa, resección de la válvula aórtica e implante de la bomba modificada, que se fijó al anillo valvular mediante puntos de sutura separados de polipropileno $2 / 0$ apoyados en pledgets de teflón (Ethibond ${ }^{\circledR}$, Ethicon) de forma similar al implante de una prótesis valvular aórtica (fig. 4). < Se hizo cierre de la aortotomía mediante la interposición de un tubo de dacrón de $30 \mathrm{~mm}$ suturado proximal y distal con sutura continua de polipropileno $05-0$ (fig. 5).

Una vez completado el cierre de la aorta, se purgó el aire de las cavidades izquierdas y se inició asistencia a flujo bajo, despinzamiento aórtico y reperfusión del corazón hasta recuperación de su ritmo propio. En caso de 
bradicardia o asistolia se apoyó al corazón con un marcapasos externo. Una vez completado el periodo de reperfusión se procedió a la salida de circulación extracorpórea y al inicio de la asistencia con la bomba a débito alto durante una hora, en la que se realizó la medición de variables hemodinámicas y ecocardiográficas.

Para mantener la hemodinamia, se apoyó al sujeto con noradrenalina en bomba de infusión continua.

Finalizada la experiencia, se procedió a la eutanasia del animal mediante la suspensión de la asistencia y la administración intravenosa de $50 \mathrm{mg} / \mathrm{kg}$ de tiopental sódico, a la extirpación del corazón y a la revisión de las cavidades cardiacas y la bomba en busca de trombos.

\section{Resultados}

Se evaluó la factibilidad técnica del implante con la técnica descrita y las complicaciones quirúrgicas que se presentan durante éste.

Se evaluaron las variables hemodinámicas (frecuencia cardiaca, presión arterial sistémica, saturación de oxígeno) y la necesidad de apoyo inotrópico durante el tiempo de asistencia.

Se hicieron evaluaciones ecocardiográficas del llenado del ventrículo izquierdo, la posición de la bomba, la apertura y el cierre de la válvula mitral durante la asistencia.

De igual forma, se evaluaron las cavidades cardiacas y la bomba postmortem para determinar el daño a las estructuras cardiacas (tracto de salida del ventrículo izquierdo; válvula mitral; ostia coronarios) y presencia de trombos.

\section{Factibilidad técnica}

Un animal falleció durante la inducción anestésica y no se pudo realizar el procedimiento. Un animal sufrió fibrilación ventricular antes de entrar en circulación extracorpórea y requirió cardioversión. Éste y los otros 6 implantes se completaron de acuerdo con la técnica descrita.

El ecocardiograma preoperatorio mostró buena función ventricular preoperatoria en todos los sujetos. El diámetro aórtico fue de 25 a $27 \mathrm{~mm}$.

El abordaje de la aorta fue difícil debido a su corta longitud. Una vez pinzada y realizada la protección del corazón, se procedió a seccionarla en forma transversa. Se observó que ambos cabos tienden a retraerse de forma importante debido a las características elásticas de las paredes del vaso.

Se resecaron los velos aórticos e instalaron suturas apoyadas en pledgets de teflón a nivel del anillo valvular. La bomba se implantó con facilidad en todos menos en un sujeto, que tenía un anillo aórtico de $25 \mathrm{~mm}$.

El cierre aórtico se hizo mediante la interposición de tubos de dacrón de $30 \mathrm{~mm}$, para permitir la llegada de los cabos de la aorta y recibir el flujo de la bomba. Debido al abordaje y falta de espacio, la sutura del tubo fue técnicamente difícil.

El tiempo de pinzamiento aórtico fue prolongado (promedio 117 minutos, rango 58 - 180), especialmente debido a la dificultad de suturar el tubo aórtico.

Hubo sangrado de las líneas de sutura en todos los sujetos, lo que impidió el cierre del tórax y la reversión de la anticoagulación. Se mantuvo la aspiración con el aspirador de la bomba de circulación extracorpórea y reinfundió el volumen aspirado por la cánula femoral para mantener la volemia.

\section{Comportamiento hemodinámico}

El electrocardiograma mostró signos de isquemia difusos en cuatro sujetos, probablemente por protección miocárdica insuficiente. Dos sujetos presentaron bradicardia severa y se apoyaron con marcapasos externo. Un sujeto no tuvo alteraciones.

Una vez cerrada la aorta se retiró el clamp e inició la asistencia con flujos progresivos de hasta $5 \mathrm{l} / \mathrm{min}$. Paralelamente se inició la ventilación mecánica y procedió al retiro de circulación extracorpórea.

Para mantener presiones arteriales adecuadas se requirió apoyo de noradrenalina en 6 de los sujetos, y sólo en uno se pudo lograr mantener la hemodinamia únicamente con el apoyo de la asistencia.

\section{Comportamiento de las estructuras cardiacas}

El ecocardiograma con asistencia mostró hipokinesia difusa del corazón en cuatro sujetos, que probablemente se debió a problemas con la protección miocárdica. Dos individuos tuvieron disfunción leve y sólo uno mantuvo una buena función del ventrículo izquierdo.

En todos los casos el llenado de las cavidades izquierdas fue adecuado.

Se observó un funcionamiento normal de la válvula mitral en 6 sujetos. En un caso se observó insuficiencia mitral grado 2 por tracción del velo anterior, que se confirmó al extirpar el corazón.

\section{Estudio postmortem}

Al explantar el corazón se comprobó la indemnidad de los ostia coronarios y de la válvula mitral, al igual que la ausencia de trombos en las cavidades cardiacas (fig. 6).

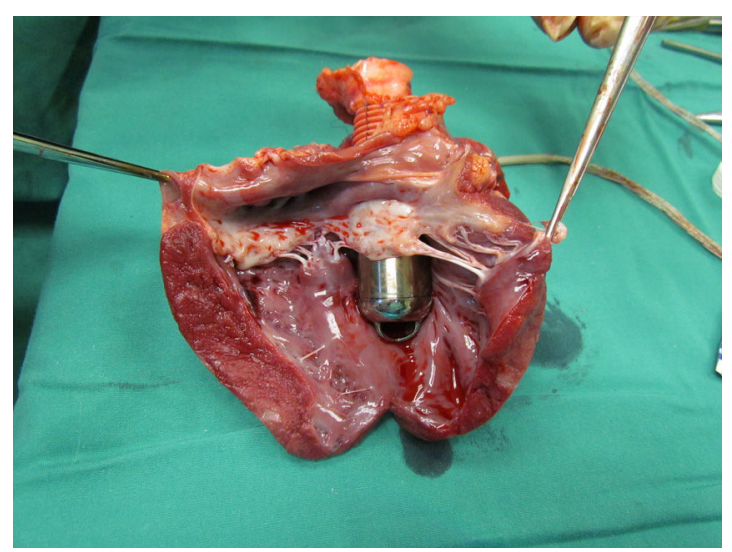

Figura 6 


\section{Discusión}

\section{La "valvo pump"}

La idea de crear una en bomba de posición de la válvula aórtica fue publicada por primera vez por Yoshinori Mitamura, en Japón, en 1991. Mitamura y sus colegas desarrollaron una bomba de flujo axial capaz de generar un flujo de $6,9 \mathrm{l} / \mathrm{min}$ y un diferencial de presión de $48 \mathrm{~mm} \mathrm{Hg}$. El tamaño de la bomba no permitió su implante en vivo ${ }^{17,18}$.

Otros equipos en China y Japón han trabajado en el desarrollo de bombas valvulares o "valvo pumps", con resultados satisfactorios en las pruebas in vitro e in vivo. Sin embargo, su tamaño aún no permite el implante en humanos ${ }^{19-21}$.

Quian-Kun, Xi y su equipo desarrollaron varios prototipos de diámetros de 25 y $23 \mathrm{~mm}$. Este último fue implantado en un cerdo de $80 \mathrm{~kg}^{23}$. El mismo equipo desarrolló una bomba de $21 \mathrm{~mm}$ de diámetro y $27 \mathrm{~g}$ de peso. Esta bomba es la más pequeña y más avanzada de su tipo. En la actualidad trabajan para mejorar su biocompatibilidad y asegurar su sostenibilidad.

No existen publicaciones de nuevos desarrollos en esta área en Occidente.

\section{Ventajas teóricas del implante intravalvular}

A diferencia del implante tradicional del Jarvik $2000^{\oplus}$ por toracotomía, el implante por esternotomía media tiene menor riesgo de sangrado, menor dolor postoperatorio y menor duración de la intervención.

La técnica es equivalente a un reemplazo de válvula aórtica, conocida por todos los cirujanos cardiacos, sin anastomosis apical o aórtica, lo que también reduciría el riesgo de sangrado. Hoy existen abordajes por miniesternotomía y minitoracotomía descritos para el reemplazo valvular aórtico, los que pueden ser utilizados para este implante con la consecuente disminución del trauma quirúrgico ${ }^{24-26}$.

El tamaño y diseño de la bomba permitiría su implante en pacientes de todos los tamaños, pues no requiere el empleo de un bolsillo, lo cual podría reducir el riesgo de infección.

La posición anatómica de la bomba permite mantener un flujo anterógrado en el tracto de salida del ventrículo izquierdo, la aorta ascendente y las arterias coronarias, lo cual podría reducir el riesgo de trombosis valvular y de embolia sistémica.

Debido a la ausencia de cicatriz apical no cambiaría la geometría ventricular y la contracción del ventrículo izquierdo sería más eficaz.

Su tamaño permitiría la implantación aórtica y pulmonar en caso de fallo biventricular.

En caso de trasplante, habría menos adherencias del pericardio, y la ausencia de un tubo protésico y la posición completamente intracardiaca de la máquina, permitirían una "cardiectomía clásica", extirpando el sistema y el corazón "en bloque". En caso de recuperación, la válvula aórtica se sustituirá por una prótesis convencional, similar a una reoperación de válvula aórtica.

\section{Desventajas del implante intravalvular}

Requiere circulación extracorpórea y pinzamiento aórtico. Sin embargo, hoy en día la circulación extracorpórea se considera de riesgo bajo ya que permite la realización de procedimientos largos y de gran complejidad en el corazón. La mayoría de las asistencias actuales, excepto el Jarvik $2000^{\oplus}$, se implantan con circulación extracorpórea.

El implante requiere el sacrificio de la válvula aórtica y el reemplazo de prótesis en caso de recuperación.

La asistencia en posición aórtica es única salida del ventrículo izquierdo y por lo tanto requiere de una absoluta fiabilidad del dispositivo. En caso de problemas técnicos se producirá una insuficiencia aórtica masiva.

Existen los inconvenientes comunes a todas las asistencias eléctricas en relación con el paso transcutáneo de la línea de energía.

\section{Nuestros resultados}

Al no existir actualmente una bomba diseñada para el implante en posición valvular se eligió el Jarvik $2000^{\circ}$, que es la única bomba existente en el mercado que está diseñada para su implante sin cánula de entrada en el ventrículo. Sin embargo, esta bomba tiene un diámetro de $27 \mathrm{~mm}$, lo que permite su paso por el anillo aórtico de animales de gran tamaño $(>100 \mathrm{~kg})$ y que son más difíciles de abordar técnicamente. Las paredes de la aorta del cerdo son más frágiles y espásticas que las del humano, y su ventrículo izquierdo es más hipertrófico. Su circulación coronaria es terminal y requiere de una protección miocárdica adecuada.

Si bien el implante pudo completarse, el modelo experimental supuso dificultades técnicas que hicieron que la cirugía fuese muy prolongada. Hubo problemas con la protección miocárdica y una duración muy larga de la circulación extracorpórea (casi 3 horas). La mala protección del ventrículo derecho obligó al uso de marcapasos para aumentar la frecuencia cardiaca, al uso de aportes de volumen para lograr un llenado adecuado de las cavidades izquierdas y al uso de vasoconstrictores para mantener una presión apropiada.

En un caso la bomba traccionó el velo mitral anterior y produjo insuficiencia mitral grado 2 .

El ecocardiograma epicárdico mostró llenado adecuado de las cavidades izquierdas y funcionamiento correcto de la válvula mitral en 5 de los 6 cerdos.

Al explantar los corazones se comprobó que en ninguno de los casos hubo obstrucción de los ostia coronarios, el anillo mitral no se deformó y la bomba dejó suficiente cavidad libre en el ventrículo para su llenado.

\section{Conclusiones}

En este modelo animal pudo completarse el implante de una bomba de flujo axial en posición valvular aórtica, pero se observaron dificultades técnicas asociadas al modelo.

La bomba fue capaz de sostener la hemodinamia del animal durante 1 hora en uno de los sujetos, y con apoyo de drogas vasoactivas en los otros. 
El implante no afectó de forma significativa el llenado ventricular izquierdo ni la movilidad y cierre de la válvula mitral.

Se debe continuar el estudio para resolver los problemas técnicos observados y aumentar progresivamente los tiempos de asistencia.

\section{¿Qué se sabe del tema?}

El implante de una bomba de flujo axial en posición valvular aórtica podría disminuir algunas de las complicaciones asociadas a estos sistemas. Sin embargo, los prototipos publicados hasta hoy están en una etapa experimental y no han sido adaptados para uso en humanos.

Por el momento, no existe una bomba diseñada para este tipo de implante y se desconocen los efectos hemodinámicos y sobre las estructuras cardiacas.

\section{¿Qué novedades aporta?}

Este estudio en un modelo animal utilizando una bomba disponible en el mercado, permitió comprobar que, a pesar de las dificultades técnicas, pudo completarse el implante de una bomba de flujo axial en posición valvular aórtica.

El anillo valvular aórtico pudo sostener la bomba.

La bomba fue capaz de sostener la hemodinamia del animal durante una hora en uno de los sujetos, y con apoyo de drogas vasoactivas en los otros.

El implante no afectó de forma significativa el llenado ventricular izquierdo, los ostia coronarios ni la movilidad y cierre de la válvula mitral.

\section{Financiación}

- Premio Thoratec 2010, otorgado por la Sociedad Francesa de Cirugía torácica y Cardiovascular. Tours, Francia.

- Association Au Service Des Personnes Malades Et Handicapees De La Poste Et De France Telecom (APCLD), a través de la Association Pour La Recherche Et L'innovation En Cardiologie (ADICARE), Paris, Francia.

- Proyecto DIUC N 211.081.028-1,0; Universidad de Concepción, Concepción, Chile.

\section{Conflicto de intereses}

Ninguno.

\section{Bibliografía}

1. Slaughter M, Rogers J, Milano C, Russell S, Conte J, Feldman D, et al. Advanced heart failure treated with continuous-flow left ventricular assist device. N Engl J Med. 2009;361:2241-51.

2. Takeda K, Takayama H, Kalesan B, Uriel N, Colombo PC, Jorde UP, et al. Long-term outcome of patients on continuous-flow left ventricular assist device support. J Thorac Cardiovasc Surg. 2014;148:1606-14.
3. Geidl L, Zrunek Ph, Deckert Z, Zimpfer D, Sandner S, Wieselthaler G, et al. Usability and safety of ventricular assist devices: human factors and design aspects. Artif Organs. 2009;33:691-5.

4. Deng M, Edwards L, Hertz M, Rowe A, Keck B, Kormos R, et al. Mechanical circulatory support device database of the International Society for Heart and Lung Transplantation: Third annual report - 2005. J Heart Lung Transplant. 2005;24:1182-7.

5. Holman W, Pamboukian S, Blood M, Tallaj J, McGiffin D, Kirklin J. Managing device infections: are we progressing or is infection an insurmountable obstacle? ASAIO Journal. 2005;51:452-5.

6. Reilly M, Wiegers S, Cucchiara A, O'Hara ML, Plappert T, Loh $E$, et al. Frequency, risk factors, and clinical outcomes of left ventricular assist device-associated ventricular thrombus. Am J Cardiol. 2000;86:1156-9.

7. Crestanelloa J, Orsinellib D, Firstenberga M, Sai-Sudhakara C. Aortic valve thrombosis after implantation of temporary left ventricular assist device. Interactive Cardiovasc Thorac Surg. 2009;8:661-2.

8. Mudd JO, Cuda JD, Halushka M, Soderlund KA, Conte JV, Russell SD. Fusion of aortic valve commissures in patients supported by a continuous axial flow left ventricular assist device. J Heart Lung Transplant. 2008;27:1269-74.

9. Rose AG, Park SJ, Bank AJ, Miller LW. Partial aortic valve fusion induced by left ventricular assist device. Ann Thorac Surg. 2000;70:1270-4.

10. Connelly JH, Abrams J, Klima T, Vaughn WK, Frazier $\mathrm{OH}$. Acquired commissural fusion of aortic valves in patients with left ventricular assist devices. J Heart Lung Transplant. 2003;22:1291-5.

11. Grimm JC, Magruder JT, Kemp CD, Shah AS. Late complications following continuous-flow left ventricular assist device implantation. Front Surg. 2015;2:42.

12. Puehler T, Ensminger S, Schoenbrodt M, Börgermann J, Rehn E, Hakim-Meibodi K, et al. Mechanical circulatory support devices as destination therapy-current evidence. Ann Cardiothorac Surg. 2014;3:513-24.

13. Mitamura Y, Nakamura H, Okamoto E, Yozu R, Kawada S, DongWook Kim. Development of the valvo pump: An axial flow pump implanted at the heart valve position. Artif Organs. 1999;23:566-71.

14. Mitamura Y, Yozu R, Tanaka T, Yamazaki K. The valvo-pump An axial, nonpulsatile blood pump. ASAIO Trans. 1991;37:M510-2.

15. Li GR, Ma WG, Zhu XD. Development of a new left ventricular assist device: the dynamic aortic valve. ASAIO J. 2001; 47:257-60.

16. Yamazaki K, Umezu M, Koyanagi H, Outa E, Ogino S, Otake Y, et al. Development of a miniature intraventricular axial flow blood pump. ASAIO J. 1993;39:M224-30.

17. Yamazaki K, Kormos RL, Litwak P, Tagusari O, Mori T, Antaki J, et al. Long-term animal experiments with an intraventricular axial flow blood pump. ASAIO J. 1997;43:M696-700.

18. Qian KX, Wang DF, Topaz S, Ru WM, Zeng P, Yuan HY, et al. Novel totally implantable trans-ventricular and cross-valvular cannular pump with rolling bearings and purge system for recovery therapy. Journal of Medical Engineering \& Technology. 2007;31:10-3.

19. Qian KX, Wang DF, Topaz S, Zeng P, Ru WM, Yuan HY, et al. World-first implantable aortic valvo pump (IAVP) with sufficient haemodynamic capacity. Journal of Medical Engineering \& Technology. 2005;6:302-4.

20. Qian KX. An implantable aortic valvo-pump for destination therapy. Cardiovasc Eng. 2006;6:41-3.

21. Qian KX, Wang DF, Topaz S, Ru WM, Zeng P, Yuan HY, et al. Worldsmallest LVAD with $27 \mathrm{~g}$ weight $21 \mathrm{~mm}$ OD and $5 \mathrm{I}$ min-1 flow with $50 \mathrm{mmHg}$ pressure increase. Journal of Medical Engineering \& Technology. 2007;31:1814.

22. directive 2010/63/EU of the european parliament and of the council of 22 September 2010 on the protection of animals used 
for scientific purposes. Official Journal of the European Union. 20.10.2010. L 276/33.

23. Ley 20.038 sobre protección de animales. 03.10.2009. Ministerio de Salud Pública. Gobierno de Chile.

24. Merk D, Lehmann S, Holzhey D, Dohmen P, Candolfi P, Misfeld $M$, et al. Minimal invasive aortic valve replacement surgery is associated with improved survival: a propensitymatched comparison. Eur J Cardio-Thorac Surg. 2014:1-7, http://dx.doi.org/10.1093/ejcts/ezu068
25. Albacker T, Blackstone E, Williams S, Gillinov M, Navia J, Roselli E, et al. Should less-invasive aortic valve replacement be avoided in patients with pulmonary dysfunction? J Thorac Cardiovasc Surg. 2014;147:355-61.

26. Bahamondes JC, Meriño G, Salman J, Silva A, Droguett JP. Miniesternotomía para cirugía valvular aórtica: Experiencia inicial en un centro cardiovascular en Chile. Rev Méd Chile. 2008;136:1141-6. 